\title{
Abnormalities of Tumor Suppressor Gene p16 in Pancreatic Carcinoma : Immunohistochemical and Genetic Findings Compared with Clinicopathological Parameters
}

\author{
K. Ohtsubo, H. Watanabe, Y. Yamaguchi, and N. Sawabu
}

Abnormalities of the tumor suppressor gene $p 16$ have been reported in a variety of human tumors, but are rare in pancreatic carcinoma (PC) except for cancer cell lines and xenografts. Moreover, their clinicopathological significance remains unknown. The purpose of this study is to examine immunohistochemical and genetic alterations of p16 in primary PC tissues, and to investigate the relationship between abnormalities of p16 and clinicopathological parameters in order to elucidate their clinicopathological significance.

We investigated p16 expression in 60 PC cases by immunohistochemistry using a monoclonal antibody clone G175-405. In addition, we analyzed genetic alterations of the p16 gene using DNA extracted from microdissected tissue of PC, by PCR-SSCP, DNA sequencing, and hypermethylation analyses using restriction enzymes. We compared the abnormalities of p16 alterations with clinicopathological parameters in order to elucidate their significance.

On immunohistochemical study, staining for the p16 protein was strongly positive in 22 (37\%) of $60 \mathrm{PC}$ cases, weakly positive in $24(40 \%)$, and negative in $14(23 \%)$. In contrast, p16 mutations were recognized in $9(15 \%)$ of the 60 PC cases. The incidence of p16 mutations was $2(9 \%)$ in 22 cases of PC with strongly positive staining, $4(17 \%)$ in 24 with weakly positive staining, and $3(21 \%)$ in 14 with negative staining. Hypermethylation of pl6 was detected in the 2 PC cases with weakly positive staining, although homozygous deletions were not found in any cases. There was no significant correlation between the expression of p16 protein and any of the clinicopathological parameters. In contrast, for PC with p16 mutation or hypermethylation, the tumor was significantly larger and the survival period significantly shorter than for PC with an intact p16 gene $(\mathrm{p}<0.05)$ (Table).

These findings suggest that $p 16$ alterations may participate in the aggressiveness of PC.

Table Correlation between clinicopathological parameters and alterations of the $p 16$ gene in pancreatic carcinomas.

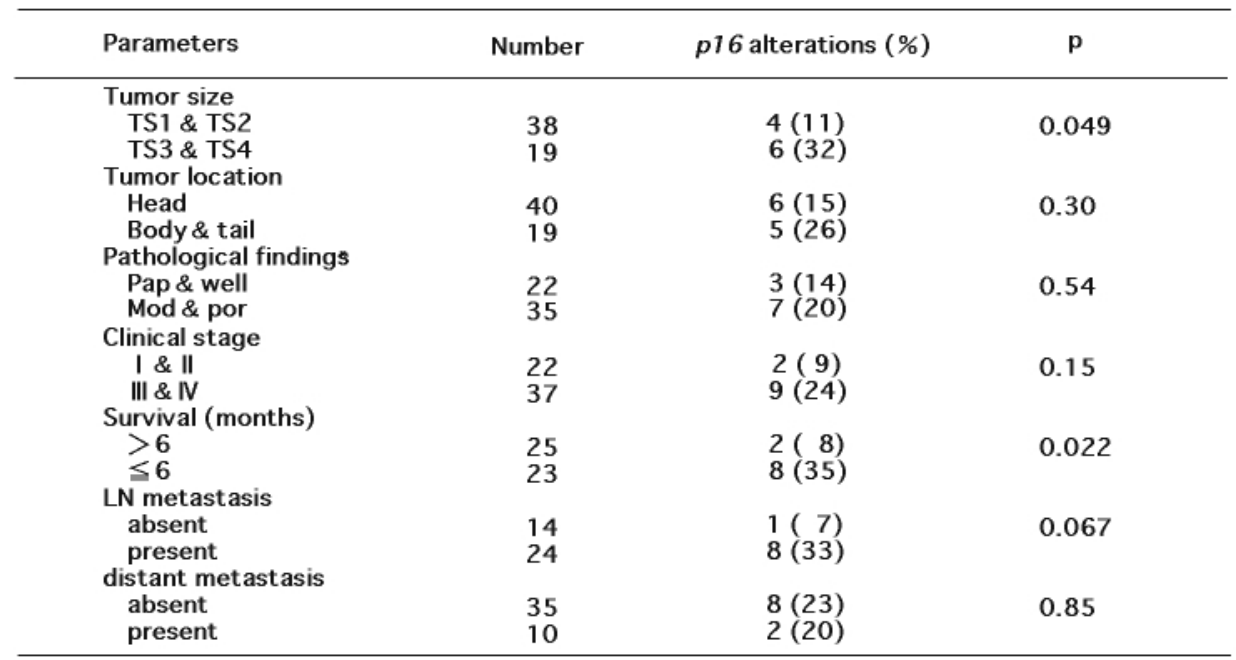

apap, papillary adenocarcinoma; well, well-differentiated adenocarcinoma; mod, moderately differentiated adenocarcinoma; por, poorly differentiated adenocarcinoma. 